\title{
Dynamic base station planning with power adaptation for green wireless cellular networks
}

\author{
Mehmet Aykut Yigitel ${ }^{1 *}$, Ozlem Durmaz Incel ${ }^{2}$ and Cem Ersoy ${ }^{1}$
}

\begin{abstract}
Energy-efficient green solutions are not only beneficial for the environment but also help to reduce the energy expenditure of the investors. Since base stations (BSs) of wireless cellular access networks are deployed to accommodate the peak-time traffic, they are underutilized most of the time. In this work, we try to save energy by both turning BSs on/off and adaptively adjusting their transmission power according to the current traffic conditions. To achieve that goal, we formulate a novel nonlinear programming model for the green dynamic BS planning (GDBP) problem to find the best possible topology which minimizes the energy consumption of the network while satisfying a certain grade of service (GoS). We derive a greedy heuristic called FastWISE to solve the formulated problem and compare our results with the results of a noncommercial optimization tool and numerous Monte Carlo experiments. It is shown that our GDBP scheme adaptively adjusts the network topology to the current traffic load and saves significant amount of energy without violating the GoS constraints, such as the probability of blocking and the coverage ratio.
\end{abstract}

Keywords: Green; Power efficiency; Green networking; Dynamic base station planning; Cellular networks; Energy awareness; FastWISE

\section{Introduction}

Along with recent increases in the energy prices, telecommunication operators started to become highly interested in energy-efficient operation. By adopting energy-aware green methods, service providers aim to decrease their operational expenses while maintaining an acceptable level of subscriber service quality. Another important motivation of green solutions is environmental awareness. Information and communication technology (ICT) industry produces $2 \%$ of the overall $\mathrm{CO}_{2}$ emission throughout the world by consuming $3 \%$ of the worldwide energy $[1,2]$. When we consider the exponential growth in data exchange [3], it is clear that the ICT sector will become one of the major $\mathrm{CO}_{2}$ emission sources within the next few decades. High energy expenditures combined with its adverse effects on environment reveal the need for novel energy-saving methods more clearly.

Since wireless cellular access networks constitute a significant portion of the ICT industry [4], it would not be

*Correspondence: aykut.yigitel@boun.edu.tr

${ }^{1}$ Computer Networks Research Laboratory, Netlab., Department of Computer Engineering, Bogazici University, Bebek, Istanbul 34342, Turkey

Full list of author information is available at the end of the article wrong to think that measures to be taken in this field can significantly contribute to make the overall communication industry greener. Although wireless cellular access networks consist of two parts, which are wired and wireless, vast majority of the energy is consumed by the wireless segment [5]. Therefore, it is considered that base stations (BSs) which are the integral part of the wireless segment are the right place to start saving energy.

Cellular access network service providers are obliged to provide a certain level of service quality regardless of time and space due to the increasing needs of subscribers. Hence, the number of deployed BSs increases rapidly parallel to the ubiquitous coverage demand of users. Figure 1 shows the base station location data of a single operator from Sydney Central Business District (CBD), Australia. Depicted BS information on the map is extracted from a website [6] which makes use of the Australian Communications and Media Authority's [7] RadCom registry. The area covered in the map is $1.5 \times 1.5 \mathrm{~km}^{2}$ and has a total of 139 BSs since Sydney CBD is one of the world's most crowded business centers and densely concentrated with skyscrapers. The reason of high BS density is that cellular network operators usually place BSs according to the peak

\section{Springer}

(c) 2014 Yigitel et al:- licensee Springer. This is an Open Access article distributed under the terms of the Creative Commons Attribution License (http://creativecommons.org/licenses/by/2.0), which permits unrestricted use, distribution, and reproduction in any medium, provided the original work is properly cited. 


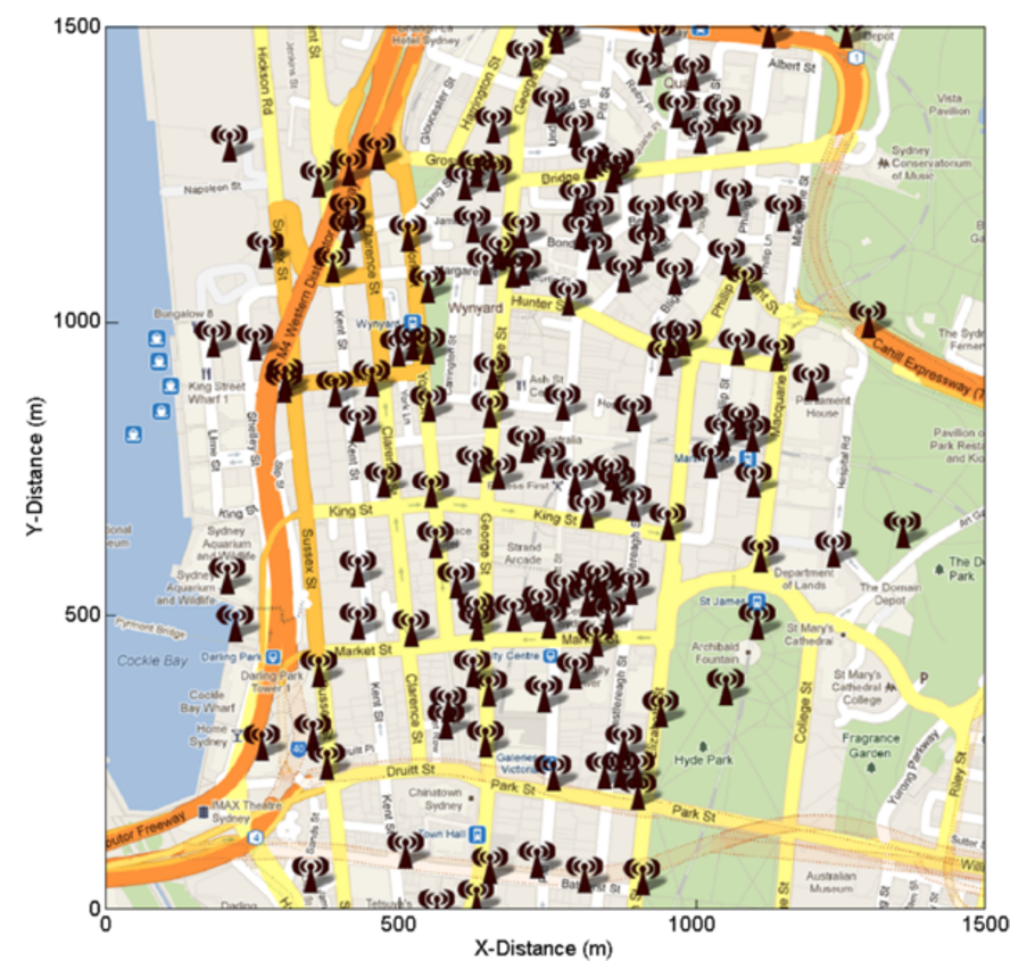

Figure 1 Base station location data of a single operator from Sydney Central Business District, Australia.

traffic conditions. However, the peak traffic mostly occurs in only a small portion of the time. A real traffic profile collected from a central BS and four neighboring BSs during 1 week can be seen in Figure 2 [8]. As expected, the traffic load decreases dramatically during the late night hours. Yet, low traffic can also be observed all day long during weekends or holidays in particular places such as business or trade centers. Therefore, infrastructures of the cellular access networks are underutilized during the nonpeak traffic periods. Hence, adoption of a green dynamic BS planning scheme can save significant amount of energy by reducing redundancy and prevent significant amount of $\mathrm{CO}_{2}$ emissions. Moreover, the reduction of active BSs also helps to mitigate the electromagnetic pollution.

In this work, we focus on saving energy by adaptively turning the BSs of wireless cellular access networks on and off according to the current traffic conditions. Moreover, we also adopt dynamic transmission power adjustment with the help of high-efficiency power amplifiers. However, the challenge is to decrease the energy expenditure while always guaranteeing a certain grade of service $(\mathrm{GoS})$ over the whole area. Therefore, we formulate a novel nonlinear programming (NLP) model for the green dynamic BS planning (GDBP) problem to find the best possible BS topology which minimizes the energy consumption while satisfying the communication demands of the users. We then propose a heuristic to solve that problem and compare our results with the results of a non-commercial optimization software and numerous Monte Carlo (MC) experiments. It is shown that our green dynamic BS planning scheme saves significant amount of energy. Although there are some studies in the literature related to the dynamic BS switching, our method differs in many aspects:

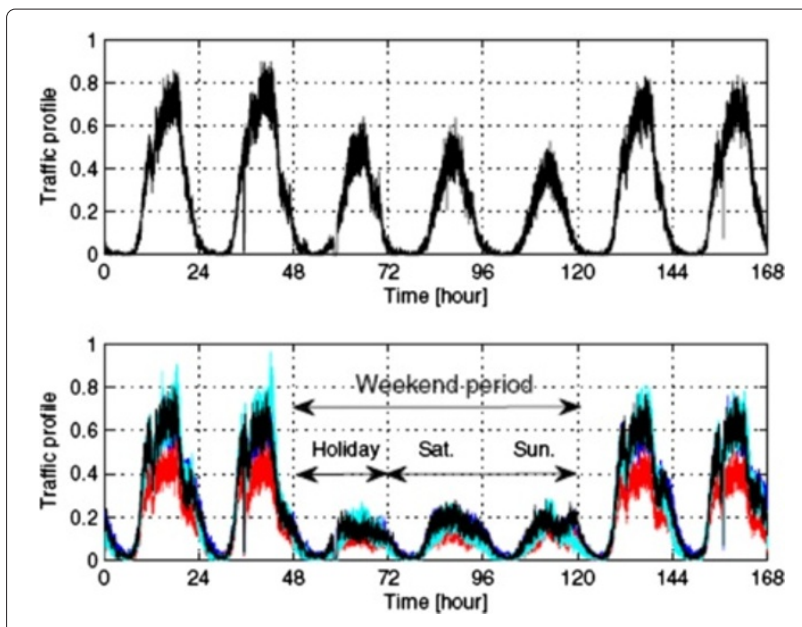

Figure 2 Normalized traffic profile of a central (top) and four neighboring (bottom) BSs during 1 week [8]. 
- Unlike most of the previous studies, we utilize the dynamic power adjustment capability of the current BSs technology by adjusting the output of the power amplifier. Using different transmission power levels (PLs), we have the opportunity to dynamically change the coverage of the BSs according to the present traffic conditions.

- Majority of the studies in the literature assume that BSs make turn on or off decisions locally by comparing their current traffic loads with a predefined threshold. In our work, we try to satisfy certain GoS requirements collectively by making system-wide decisions throughout the whole network.

- The BS on/off transitions are taken into account in order to minimize the additional overhead introduced by frequent topology changes such as BS initialization, user association, and handover.

- We justify our proposed methods by applying them to real-life-scale scenarios rather than small-scale test cases.

- A detailed integer NLP model is formulated for the GDBP problem and solved by a non-commercial optimization tool. By using the derived programming model, optimum results can be obtained from the optimization tools for the small instances of the problem. In order to show the significance of the results, a very large number of $\mathrm{MC}$ experiments are also conducted.

- A fast and effective heuristic called FastWISE is proposed for solving large instances of the GDBP problem.

The rest of the paper is organized as follows: In Section 2, existing green network planning methods in the literature are surveyed. Section 3 elaborates the proper application areas of the GDBP, assumptions, and problem formulation. The proposed GDBP algorithm is explained in Section 4. An example application scenario, details of the system parameters, and comparative performance analysis of the proposed methods are presented in Section 5. Finally, Section 6 draws the conclusions and provides possible directions for future research.

\section{Related works}

Optimal energy-saving expressions as a function of the daily traffic profile are given in [9]. In order to apply the derived expressions in real-life scenarios, Marsan et al. propose a set of realistic regular cell topologies where each of these configurations has a specific energy-saving ratio by turning three out of four or eight out of nine BSs off. They compared the proposed topology configurations in terms of energy saving by using a realistic traffic pattern collected from a wired broadband service provider.
In [10], Son et al. propose a theoretical framework for two problems which are dynamic BS operation and user association. Rather than solving those two problems jointly, they decompose it into two subproblems and try to handle them separately. For the user association problem, they offer a distributed algorithm composed of a mobile terminal part and a base station part. For the dynamic BS operation problem, they derive two greedy BS turn on/off algorithms. They also provide three more heuristics which consider the inter-BS distance and BS utilization as decision metrics.

Zhou et al. [11] propose a centralized and a decentralized BS energy-saving algorithms. In the centralized one, it is assumed that a single entity has all channel and traffic information required to decide turning BSs on or off. In the decentralized algorithm, a BS association rule is derived for users to concentrate the traffic load on some particular BSs so that the remaining redundant ones can be turned off easily. Another distributed BS switch on/off algorithm was proposed in [8]. The analysis provided in this work shows that the mean and variance of the traffic profile along with the BS density are the dominant factors that determine the amount of energy saving.

Recently, Oh et al. [12] propose an algorithm called SWES along with three other versions of it for BS switching on/off. They introduce the notion of network-impact which considers the effect of BS transitions on the neighboring BSs in terms of traffic load and try to find solutions which have minimum effect on the network. It is shown that, according to the test case results conducted with real-life topology and traffic data, their algorithms can achieve energy savings up to $80 \%$.

In [13], Saker et al. assume all BSs have a set of resources which can be activated and deactivated according to the traffic load where the term resource refers to available transmitters for GSM and carriers for 3G. Their method is based on use-as-required principle where communication resources of the BSs are activated as the cell load increases and deactivated as the load decreases. They bring two practical implementation issues into attention which are guard period and ping-pong effect along with their impact on energy consumption of the network. Similarly, novel network dimming methods are proposed in [14] rather than completely turning the BS off. Three basic network dimming methods are derived for coverage, frequency, and service dimming. A joint optimization problem is formulated for both frequency and service dimming, and it is shown that reducing resource usage is a promising technique for energy saving.

Since it is very common that more than one cellular access network operator offers service over the same area, cooperative management of the two separate networks was proposed in [15] to save energy by switching off one of the two during low traffic. Although this technique 
seems to be promising, cellular network operators should closely cooperate with each other and act as a one from the subscriber's point of view.

In the literature, there are quite few examples which consider the dynamic cell size adjustment in order to reduce energy consumption. Among them, Niu et al. [16] introduce the cell zooming concept for energy saving to adaptively adjust the size of the cells according to the current traffic load. In their work, a cell zooming server which is a virtual entity in the network controls the procedure of cell zooming. The cell zooming server collects the information such as the traffic load, channel conditions, and user requirements and then analyzes whether there are opportunities for cell zooming or not. They also proposed centralized and distributed versions of user association algorithms for cell zooming.

Another work considering variable cell sizes for energy saving is [17]. In this work, Bhaumik et al. consider two types of BSs which are subsidiary BSs with low transmit power and umbrella BSs with high transmit power. They propose a self-operating network by adaptively turning subsidiary and umbrella BSs on and off according to the current traffic demands. Similarly, Kokkinogenis et al. [18] assume a cellular network consisting of micro and macro BSs where micro BSs have the ability of being switched on/off while macro BSs can iteratively adjust their transmission power until the required QoS is achieved. They propose a static centralized, a dynamic distributed, and a hybrid topology management schemes to reduce the overall energy consumption of the network while satisfying certain QoS requirements. Chiaraviglio et al. [19] propose a novel approach to save energy in UMTS networks by reducing the number of active access devices when they are underutilized. Authors derive two models for both circuit-switched and packet-switched services separately for quantification of possible energy savings. For further information, readers may refer to the green cellular network survey in [20].

Although we are utilizing the dynamic cell size adjustment strategy in our work, we assume a set of predefined cell sizes unlike other related works. Hence, we are able to formulate a mathematical model for the GDBP problem and solve it with an optimization tool. We also take the overhead of BS on/off transitions into account and try to minimize the total number of transitions. Moreover, we defined a fast and effective heuristic to solve the larger realistic size problems and justified our results with real-life-scale scenarios rather than small test cases.

\section{System model}

Before going into detail, possible application areas of the GDBP along with their advantages and disadvantages are investigated from the green networking perspective.

\subsection{Where should GDBP be applied?}

As we mentioned before, the primary objective of GDBP is to save energy while satisfying a certain level of service quality. Hence, there must be excess energy consumption in order to benefit from GDBP properly. If the energy is already being used effectively, applying an energy-saving method will be no more than unnecessarily increasing the complexity. Crowded urban areas with high BS densities are the most suitable places for GDBP rather than suburban or rural areas. However, each urban area has its own traffic pattern which directly determines the efficiency of the GDBP. Therefore, we categorized urban areas into four distinct regions and commented on those regions whether GDBP should be applied or not.

- Town centers (business). Business, trade, or industrial areas as well as commercial centers can be considered in this class. The user density, hence the offered traffic load, is quite high in these places during the daytime. However, the user density and the traffic load drop sharply during the nighttime since most of the business and commercial areas are closed. Moreover, low traffic profiles continue all day long during weekends and holidays. Therefore, a significant change in the traffic profile occurs throughout the day and week which makes business town centers the most suitable places for GDBP to be applied.

- Town centers (entertainment). This kind of places include shopping and exhibition centers, tourist attraction points, museums, and concert halls. Although the traffic profile of entertainment and business town centers follow a similar pattern, they differ during weekends and holidays. Entertainment town centers are also highly preferred during weekends and holidays, even more than weekdays. However, the temporal change throughout the day does not happen to be as much as business town centers. Therefore, entertainment town centers are our secondary target for energy saving.

- Residential areas. These regions are mostly occupied by houses, schools, hospitals, and small commercial shops such as grocery stores. User density increases here in the evening for sure. However, it would not be true to say that there is no traffic at all during the day time. Individuals such as pensioners, housekeepers, or children spend most of their time within the territory of their houses. Although the traffic load changes in residential areas within the day, it is not as explicit as in town centers.

- Seasonal tourism centers. In seasonal tourism centers, there happens to be two colossal changes in user density throughout the year. Sunny seasides are filled up with tourists during summer, whereas snowy ski centers are very crowded during winter. However, 
most of the wireless network operators simply deploy mobile BSs to those areas in order to meet the high season requirements. Since using mobile BSs is a kind of dynamic planning itself, it can be considered as a broader and more systematic approach to GDBP including additional capabilities of BS installment and replacement.

In summary, the application site should have at least two important features in order to fully benefit from GDBP: (i) unbalanced temporal distribution of the traffic load and (ii) high BS density.

\subsection{Assumptions}

A BS can be up or down depending on the current traffic conditions in our work. When it is turned on, the total power consumption of the BS is the combination of two components [21]: (i) core power and (ii) transmission power. The BS core power consumption (such as air conditioning, signal processing) is assumed to be fixed regardless of the traffic load. However, the transmission power is adaptively adjusted to the current traffic conditions. A set of transmission power levels need to be defined according to the application requirements and the capabilities of the BS equipment in use. Each BS can select a certain PL for transmission and cannot change it during that particular time slot. Since it is not practical to model a huge number of subscribers individually, we assumed users are placed as chunks like group of workers in a floor of a building or customers waiting in a bank office.

\subsection{Problem formulation}

In order to solve the problem by classical optimization tools, we need to first put the GDBP problem into a mathematical form. In this section, we formulated our problem by using two different objective functions. The first one minimizes the total energy consumption, while the second one additionally minimizes the BS on/off transitions in order to reduce the amount of topology changes. Hence, the overhead caused by frequent topology changes, such as BS initialization, user association, and handover, can be minimized.

\subsubsection{Plain GDBP}

Our formulation consists of three parts. The first part contains the constant parameters given by our sample application scenario. The second part is the model variables which will be determined by the solver, and the last part is the problem itself.

\section{Parameters}
$N^{B} \quad$ : Number of BSs
$N^{P} \quad$ : Number of PLs
$N^{U} \quad$ : Number of user chunks (UCs)

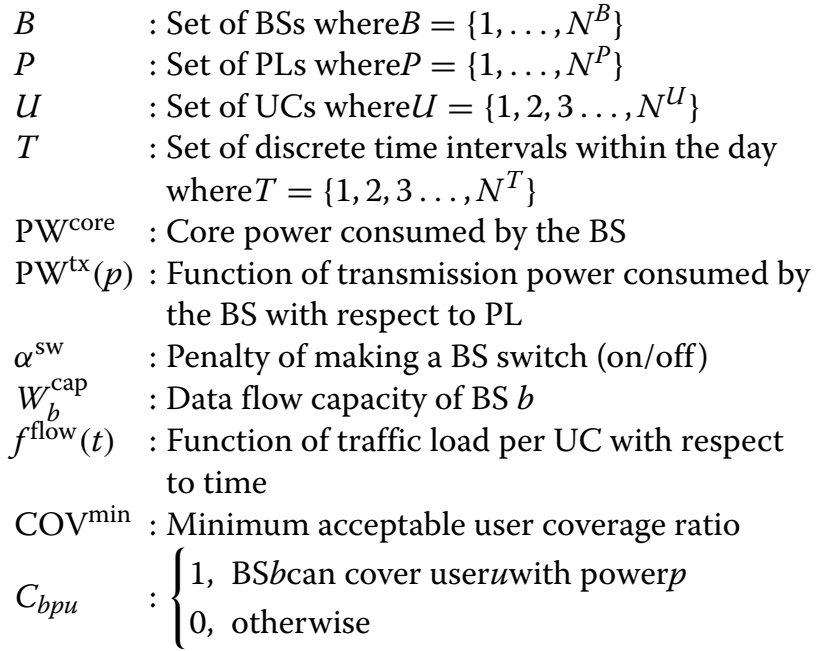

Model variables

$$
\begin{aligned}
& O_{b t}= \begin{cases}1, & \text { BS } b \text { is up at time } t \\
0, & \text { otherwise }\end{cases} \\
& A_{b p t}= \begin{cases}1, & \text { BS } b \text { transmits with power } p \text { at time } t \\
0, & \text { otherwise }\end{cases} \\
& M_{u b t}= \begin{cases}1, & \text { UC } u \text { selects BS } b \text { at time } t \\
0, & \text { otherwise }\end{cases}
\end{aligned}
$$

Dummy variables

$$
\begin{aligned}
S_{u b t} & = \begin{cases}1, & \mathrm{UC} u \text { is served by BS } b \text { at time } t \\
0, & \text { otherwise }\end{cases} \\
& =\sum_{p \in P} O_{b t} C_{b p u} M_{u b t} A_{b p t} \forall u \in U, \forall b \in B, \forall t \in T \\
N_{t}^{c} & =\text { Number of covered UCs at time } t \\
& =\sum_{u \in U} \sum_{b \in B} S_{u b t} \quad \forall t \in T \\
N^{\mathrm{sw}} & =\text { Number of BS switches (on/off) during } 24 \mathrm{~h} \\
& =\sum_{b \in B} \sum_{t \in T}\left(O_{b t} \oplus O_{b\left((t+1) \bmod N^{T}\right)}\right)
\end{aligned}
$$

The GDBP problem

$$
\begin{aligned}
& \min \sum_{b \in B} \sum_{p \in P} \sum_{t \in T} O_{b t}\left(\mathrm{PW}^{\text {core }}+A_{b p t} \mathrm{PW}^{\mathrm{tx}}(p)\right) \\
& \text { s.t. } \\
& \sum_{p \in P} A_{b p t}=1 \quad \forall b \in B, \forall t \in T
\end{aligned}
$$




$$
\begin{aligned}
& \sum_{b \in B} M_{u b t}=1 \quad \forall u \in U, \forall t \in T \\
& \sum_{u \in U} S_{u b t} f^{\text {flow }}(t) \leq W_{b}^{\text {cap }} \quad \forall b \in B, \forall t \in T \\
& \frac{N_{t}^{c}}{N^{U}} \leq \mathrm{COV}^{\text {min }} \quad \forall t \in T
\end{aligned}
$$

As we mentioned before, the ultimate goal of our first objective function in (1) is to minimize the energy power consumption of the network. The constraint in (2) makes sure that a BS operates at a single transmission power level at the same time, and (3) is responsible for a user being served by a single BS at a particular instant. (4) and (5) ensure that the resulting energy-efficient topology does not violate the capacity constraint of the BSs and provides the required coverage ratio over the area, respectively. By not violating the capacity constraints of the BS, it is also assured that subscribers receive an acceptable service quality.

\subsubsection{GDBP with BS transition overhead}

In this section, we are taking the BS transitions into account in order to minimize the additional overhead introduced by frequent topology changes such as BS initialization, user association, and handover [22]. Among them, handling the handovers is the most crucial one since it directly affects the service quality of the subscribers. Except well-known problems inherited from conventional handover procedures, another challenging issue is to handover a group of subscribers at the same time when a serving BS is turned off. There has been some research effort on group handover techniques [23,24], and most of them target the passengers traveling on public transportation vehicles such as buses and trains. Majority of the group handover schemes require to predict the handover and make necessary preparations before starting the handover procedure itself. In our case, the central control entity, which decides and implements the network topology changes, may do the necessary control signaling and inform the neighboring BSs about the possible group handover before shutting a BSs down. Also, a possible BS transition and handover procedure is discussed in [12].

In order to minimize the side effects of topology changes, we used a second objective function in (6) which minimizes the BS on/off switches in addition to the overall power consumption. The BS switch penalty $\alpha^{\mathrm{sw}}$ controls the power consumption vs. BS transition overhead trade-off. Thus, network operators have the chance to fine tune the objective function according to their priorities. The effect of this parameter is further investigated in Section 5.3.

$$
\min \sum_{b \in B} \sum_{p \in P} \sum_{t \in T} O_{b t}\left(\mathrm{PW}^{\text {core }}+A_{b p t} \mathrm{PW}^{\mathrm{tx}}(p)\right)+\alpha^{\mathrm{sw}} N^{\mathrm{sw}}
$$

Although we put the GDBP problem into a mathematical form, it is still a challenging task to solve it with the optimization tools since we use real-life-scale test scenarios for performance evaluation. Furthermore, nonlinearity of the problem also increases its complexity and yields to longer run times. Therefore, we propose a fast heuristic to solve large-scale instances within acceptable time durations.

\section{Green dynamic BS planning algorithm}

In this section, we derive a heuristic called FastWISE for the GDBP problem which consists of three phases.

Additional variables used in FastWISE

$\mathrm{OCA}^{\text {cur }}$ : Overlapping coverage area of the current BS

$\mathrm{OCA}^{\mathrm{max}}$ : Maximum allowed overlapping coverage area in order to turn a BS on during initialization phase

$B^{\text {off }} \quad:$ Set of currently turned off BSs

$W^{\text {cur }}$ : Traffic load of the current BS

$B^{\text {high }} \quad$ : Set of turned on BSs having $W^{\text {cur }} \geq W^{\text {cap }}$ (users served by those BSs most likely to suffer high blocking probabilities)

CUE : Covered $^{\mathrm{a}}$ user per energy ratio of the current BS when it is turned on

$\mathrm{COV}^{\text {cur }}$ : Current user coverage ratio of the network 
FastWISE starts with the initialization phase. In this phase, FastWISE visits all BSs and activates the ones which have smaller overlapping coverage than a predefined threshold with the maximum possible transmission power level. By doing this, FastWISE tries to use BSs with higher transmission power levels without violating the capacity constraints in order to give energy-saving opportunities to neighboring BSs. Therefore, a preliminary coverage is provided at the end of this phase. FastWISE continues with the iteration phase. The aim of this phase is to make incremental improvements at each step on top of the preliminary coverage produced by the initialization phase until a target coverage ratio throughout the network is achieved. Initially, a covered user per energy (CUE) ratio is calculated for each inactive BS for each power level. This ratio implies the number of additional covered users per unit energy if that particular BS is turned on. As long as the desired coverage ratio is not achieved, the BS having the highest CUE ratio is simply turned on. Unlike the initialization, the iteration phase tries to maximize the energy utilization without making any capacity constraint checks. However, this may yield to overloaded BSs which in turn cause higher call blocking probabilities. Therefore, the third and the last step are required to validate that the traffic capacity constraints are met for all serving BSs, which is the validation phase. In this last part of the heuristic, all serving BSs are visited and a list of neighboring BSs is created for all overloaded ones. In order to share the load of the overloaded BSs, starting from the closest one, neighboring BSs in the list are simply activated with the minimum transmission power level until the offered traffic load drops below its capacity. At the end of this phase, FastWISE ensures that all serving BSs are operating well below their capacities.

\section{Application scenario and performance evaluation}

\subsection{Application scenario and parameters}

In order to model the unbalanced temporal distribution of the load created by mobile users, we are assuming a sinusoidal pattern throughout the day resembling the reallife traffic pattern given in Figure 2 and the many other measurement studies presented in $[8,14,25]$. However, the traffic profile does not strictly have to follow the shape of a sine wave. For the GDBP, reasonable amount of temporal traffic fluctuations through out the day will create a margin for energy saving. Although we have a certain traffic profile assumption, it is still possible to engineer the shape of that profile up to some extent. For example, the nighttime traffic load may not be as low as we expect or the peak-time traffic may not even get close to $100 \%$ utilization in some particular places. Therefore, we introduce a lower and a higher bound for the traffic load rather than assuming a regular sinusoidal wave ranging between $0 \%$ and $100 \%$ utilization. In fact, when we introduce those lower/higher bounds, we practically define the height and offset of the sinusoidal wave itself. Hence, they together define how the traffic load changes throughout the day. The final and vital parameter to construct the traffic profile is the time slice in which the traffic load reaches its peak. With this parameter, we can shift the sinusoidal wave in time domain until it fits the traffic profile of a region of interest. The traffic function is defined as

$$
\begin{gathered}
w_{\text {height }}=\frac{\rho_{\max }-\rho_{\min }}{2} \\
w_{\text {offset }}=\frac{\rho_{\max }+\rho_{\min }}{2} \\
\rho(t)=w_{\text {height }} \cos \left(2 \pi \frac{t-t_{p}}{N_{T}}\right)+w_{\text {offset }}
\end{gathered}
$$

where $\rho_{\min }$ and $\rho_{\max }$ are the minimum and the maximum traffic loads throughout the day, $w_{\text {height }}$ and $w_{\text {offset }}$ are the height and offset of the sinusoidal traffic wave, and $t_{p}$ is the time slice in which the traffic load has its peak. An example traffic profile created by (7) can be seen in Figure 3.

We adopt three distinct transmission PLs for BSs which we believe is not irrational when the current state of the $\mathrm{BS}$ manufacturing technology is considered. If a BS is up, then it is transmitting with one of $\mathrm{PL}_{n}$ where $n \in\{1,2,3\}$. When we change the transmit power of a BS, we subsequently change its coverage range. Since all of our test area exhibits the same terrain feature (urban), a single propagation model is used throughout the whole area. However, in case of need, the test area may be partitioned into different terrain features and other propagation models can be incorporated for those specific portions of the coverage area. We assumed perfect free-space path loss for calculating the omnidirectional coverage ranges. When we

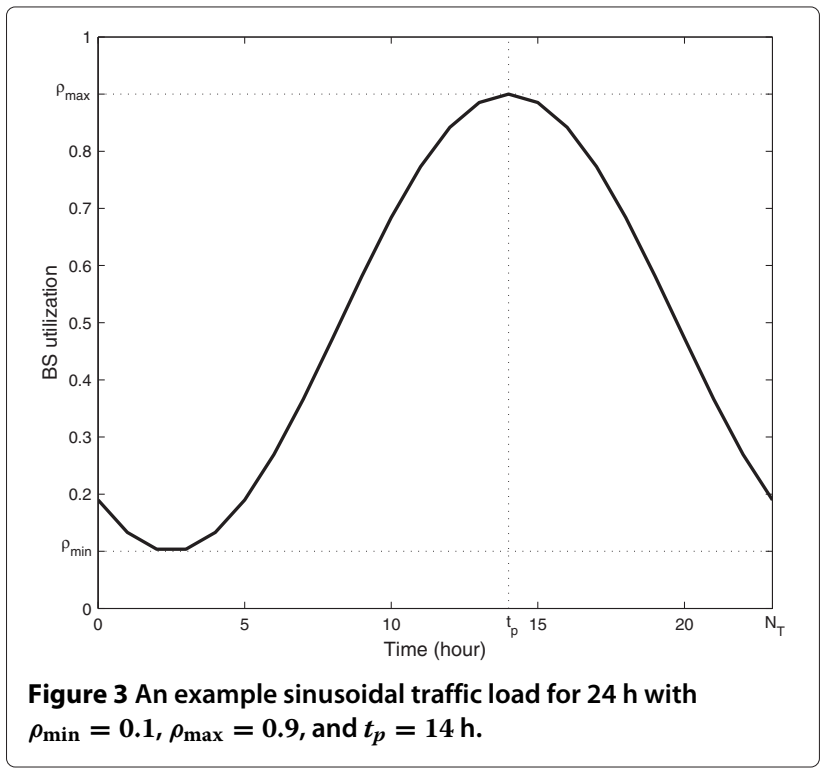




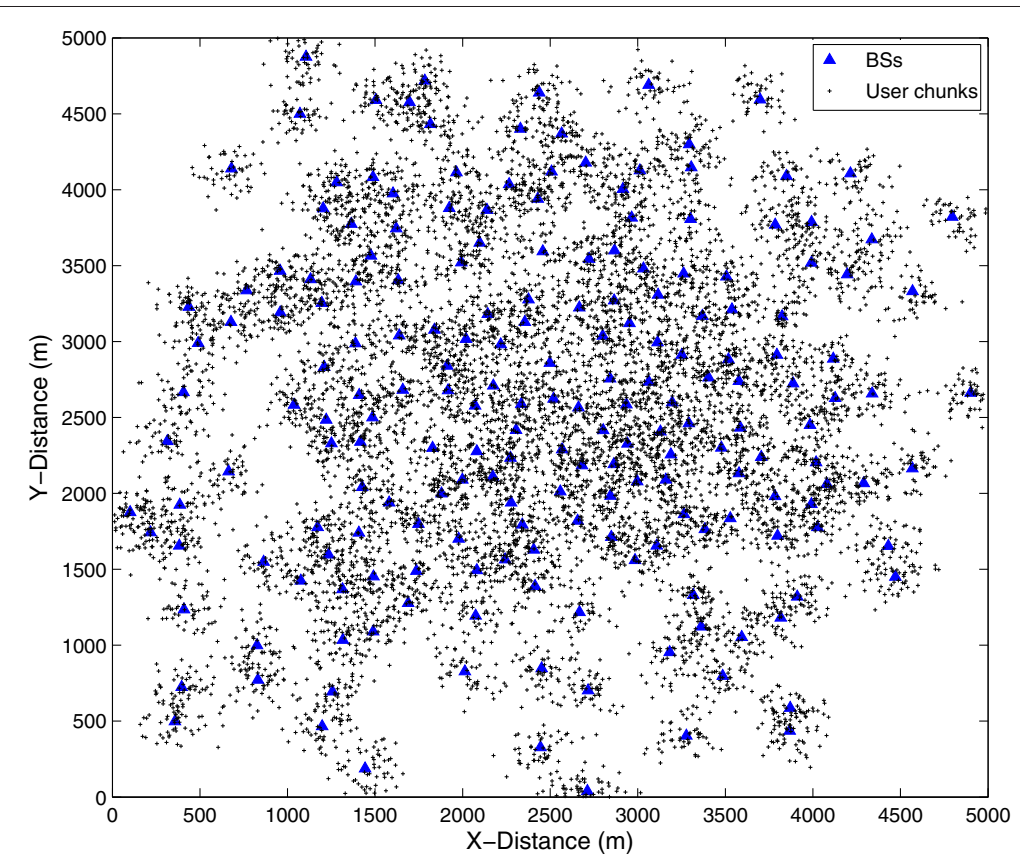

Figure 4 Sample deployment configuration with 10,000 UCs (one million users) and 200 BSs in a 5 x 5 km² area.

fixed the signal frequency, free-space path loss becomes proportional to the square of the distance between the transmitter and receiver. However, all propagation models can be used with our problem formulation according to the wireless channel conditions in the coverage area.

Although our model can accommodate BSs with different traffic flow capacities, we assume all BSs are identical and have the same capacity. Both user chunks and BS locations follow Gaussian distributions where BSs are centered in the middle of the area and user chunks are centered around the BSs. However, two BSs cannot be closer than the minimum inter-BS distance (MIBD) to each other.

In order to make proper assessments of the proposed methods, it is required to create a test environment as close to real-life conditions as possible. Therefore, we envisioned a densely populated (one million subscribers) business center as advised in Section 3.1 which is covering an area of $5 \times 5 \mathrm{~km}^{2}$. We assume that the traffic load follows the same pattern in Figure 2 and there are 200 BSs deployed to accommodate the peak-time traffic. A sample deployment configuration used for performance evaluation is given in Figure 4. As GoS metrics, the network should provide the maximum of $10^{-2}$ blocking probability [26] and cover at least $99 \%$ of the area at all times. Important parameters used in the sample application scenario are summarized in Table 1 . For the sake of variance control, 10 different test cases are generated and average of the results is presented.

\subsection{Experiment methodology}

Performance of FastWISE is evaluated by using reallife-scale test cases and compared with the results of a NLP tool [27]. Also, MC experiments are used by generating a large set of random solutions to investigate

\section{Table 1 Scenario parameters}

\begin{tabular}{ll}
\hline Parameter & Value \\
\hline Coverage area & $5 \times 5 \mathrm{~km}^{2}$ \\
Number of BSs & 200 \\
Number of UCs & 10,000 \\
Chunk size & $100 \mathrm{users}$ \\
BS location std. dev. & $1,000 \mathrm{~m}$ \\
User location std. dev. & $100 \mathrm{~m}$ \\
MIBD & $150 \mathrm{~m}$ \\
BS core power & $150 \mathrm{~W}$ \\
Number of PLs & 3 \\
BS transmission PLs & 30,90, and 270 W \\
BS coverage distances & 300,520, and $900 \mathrm{~m}$ \\
BS capacity & 66 Erlang \\
Max. prob. of blocking & $10^{-2}$ \\
Average call duration & $30 \mathrm{~s}$ \\
Average call arrival rate & 10 calls/day/user \\
Number of time slots within a day & 24 \\
Min. acceptable coverage ratio & $99 \%$ \\
Penalty of a BS switch & $0,75,300$, and 1,500 \\
\hline
\end{tabular}


Table 2 Comparison of average run times

\begin{tabular}{|c|c|c|c|}
\hline \multicolumn{2}{|c|}{ FastWISE } & \multicolumn{2}{|c|}{ NLP } \\
\hline Phase & Run time & Time slot & Run time \\
\hline \multirow{4}{*}{ Initialization } & \multirow{4}{*}{$4 \min 3 s$} & 1 and 6 and 24 & $296 \min$ \\
\hline & & 2 and 5 & $268 \mathrm{~min}$ \\
\hline & & 3 and 4 & $253 \mathrm{~min}$ \\
\hline & & 7 and 23 & $312 \mathrm{~min}$ \\
\hline \multirow{4}{*}{ Iteration } & \multirow{4}{*}{$65 \min 46 s$} & 8 and 22 & $423 \mathrm{~min}$ \\
\hline & & 9 and 21 & $456 \mathrm{~min}$ \\
\hline & & 10 and 20 & $501 \mathrm{~min}$ \\
\hline & & 11 and 19 & $517 \min$ \\
\hline \multirow{4}{*}{ Validation } & \multirow{4}{*}{$12 \mathrm{~s}$} & 12 and 18 & $538 \mathrm{~min}$ \\
\hline & & 13 and 17 & $542 \mathrm{~min}$ \\
\hline & & 14 and 16 & $535 \mathrm{~min}$ \\
\hline & & 15 & $548 \mathrm{~min}$ \\
\hline Total & $70 \min 1 \mathrm{~s}$ & Total & $5,189 \mathrm{~min}$ \\
\hline
\end{tabular}

the statistical quality of the FastWISE results. However, the initial results of fully random MC experiments were mostly unfeasible and too poor to be compared with other results. In order to obtain more challenging results, we changed the random solution generation method by assigning different probabilities of drawing to each case and call it $\mathrm{MC}^{*}$. By this way, we created 100,000 biased samples which contain much more feasible results than the fully random MC method. The idea behind $M C^{*}$ is to generate more suitable topology instances by taking the current traffic load into account. For example, $\mathrm{MC}^{*}$ turns more BSs on if the traffic load is high and less BSs if the traffic load is low. Similarly, $\mathrm{MC}^{*}$ favors higher power levels for the activated BSs during low traffic conditions to create a margin for neighboring BSs to save energy. Thus, $\mathrm{MC}^{*}$ creates more feasible solutions than the plain $\mathrm{MC}$ and gives us the chance to make better assessment of the proposed techniques.

We modeled the problem with AMPL (A Modeling Language for Mathematical Programming) [28] and used a non-commercial nonlinear optimization tool called BONMIN (Basic Open-Source Nonlinear Mixed Integer Programming) [27]. However, although we used a very powerful computer, it was not possible to solve the problem as a whole due to high space and computational complexity. Therefore, we decompose the problem into smaller parts. For Plain GDBP, we solve each time slot separately and add them up to find the objective function given in (1). We approach the second problem similarly, but this time, we feed the results of the previous slot as an input to the next one in order to compute the objective function given in (6).

\subsection{Performance evaluation}

Before proceeding to the comparative performance evaluation, we find it useful to start with examining run times. Average run times of FastWISE and NLP which are collected from a powerful computer with 4 hexa-core Xeon x5650 $2.67 \mathrm{GHz}$ processors and 24 GB of memory are given in Table 2. For FastWISE, the iteration is observed to be the most time-consuming phase as expected since small improvements are done until a target coverage ratio is achieved, though the overall execution time of the FastWISE can be easily considered as acceptable. On the other hand, NLP takes longer time to find feasible solutions than FastWISE, and this time increases parallel to the offered traffic load. In Table 2, 24 time slots are reduced to 12 since some of them have the same amount of traffic load due to the sinusoidal traffic profile. It takes close to an average of 4 days for the NLP tool to find a solution for one instance.

The comparative power consumptions throughout a day are given in Figure 5. If none of the green techniques is applied to the network, the power consumption does not change throughout the day regardless of the varying traffic load. ${ }^{c}$ Although some amount of power can be preserved with MC*, it is clear that both FastWISE and NLP perform better in terms of the power consumption. NLP outperforms FastWISE in light traffic conditions, while the opposite is valid under heavily loaded conditions. Due to large scale of the test scenario and high computational complexity of the proposed NLP, we set a maximum

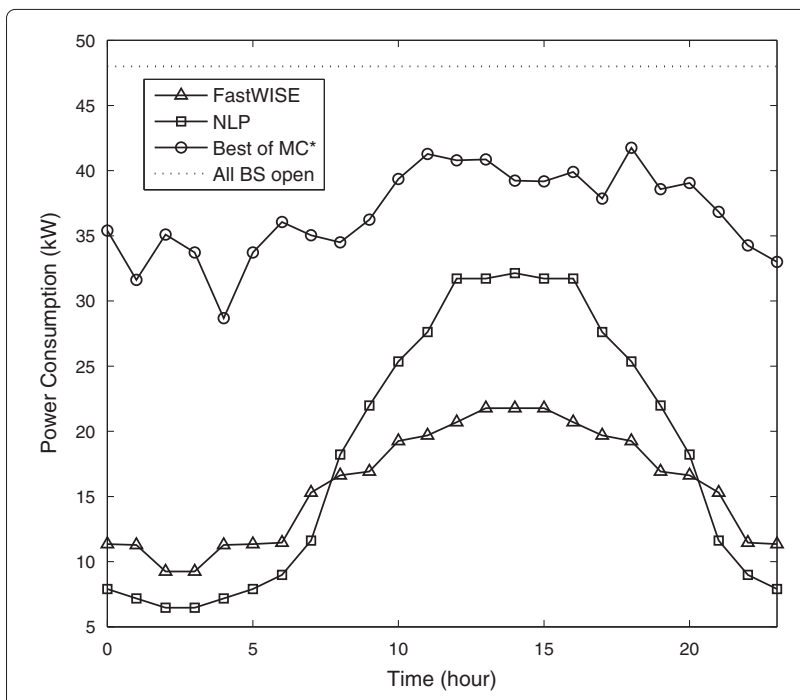

Figure 5 Comparative power consumption throughout a day. 
Table 3 Comparative energy cost saving

\begin{tabular}{lccc}
\hline & Daily (\$) & Monthly (\$) & Annual (\$) \\
\hline FastWISE & 168 & 5,043 & 60,521 \\
NLP & 143 & 4,317 & 51,809 \\
MC* & 55 & 1,654 & 19,857 \\
\hline
\end{tabular}

iteration limit on the optimization software in order to bound the run times. It returns the best possible solution found within the given number of branch-and-bound iterations.

In Table 3, daily, monthly, and annual energy cost savings are given. The electricity prices for peak $(2 \mathrm{pm}$ to $8 \mathrm{pm}$ ), shoulder ( $7 \mathrm{am}$ to $2 \mathrm{pm}$, and $8 \mathrm{pm}$ to $10 \mathrm{pm}$ ) and off-peak (all other times) times are 44.11, 18.7, and 10.34 cents $/ \mathrm{kWh}$, respectively, in compliance with the EnergyAustralia [29], one of Australia's largest electricity retailers. When the given figures in Table 3 are scaled for the whole country, it is clear that GDBP can dramatically decrease the energy expenditures of the service providers, possibly a few millions of dollars per year, which constitutes the largest portion of the operational expenses.

In Figure 6, the probability distribution of feasible MC experiments is given with a fitted Gaussian distribution. When averaged results of FastWISE and NLP are given in the same figure compared with the results of the MC experiments, it is quite certain that they are statistically significantly better. In other words, it is nearly impossible to generate results with $\mathrm{MC}$ experiments as power efficient as the ones with FastWISE and NLP.

Figures 7 and 8 evaluate the GDBP with BS transition overhead introduced in Section 3.3.2. Figure 7 depicts the effect of $\alpha^{\mathrm{sw}}$ on the objective function given in (6). When we set $\alpha^{\mathrm{sw}}=0$, the objective function reduces to plain GDBP given in (1). For its maximum value, we set $\alpha^{\mathrm{sw}}=$ 1,500 . In this case, BS transition penalty in the objective function dominates the transmission power consumption, and the network tends to keep its current topology rather than adapt to the changing traffic conditions. As the BS switch penalty increases, the objective function value also increases. When we set the switch penalty to higher values, the optimization tool does not turn off the redundant BSs as long as the resulting energy saving is smaller than the introduced transition overhead. Therefore, the topology is adjusted by turning a large number of BSs on or off for higher transition penalties. As a result, the objection function graph takes a more zigzag-like shape for higher penalties, while it is smoother for lower values of $\alpha^{\mathrm{sw}}$.

When a switching penalty is introduced in the objective function, the number of BS transitions dramatically decreases as seen in Figure 8. This figure depicts the cumulative sum of BS transitions for different $\alpha^{\mathrm{sw}}$ values. The total number of BS transitions throughout the day is reduced by $52 \%, 89 \%$, and $93 \%$ for $\alpha^{\mathrm{sw}}=75,300$, and 1,500 , respectively. Hence, the additional overhead introduced by frequent topology changes is significantly reduced. However, as the BS switch penalty gets higher, the flexibility of the GDBP decreases which yields to less

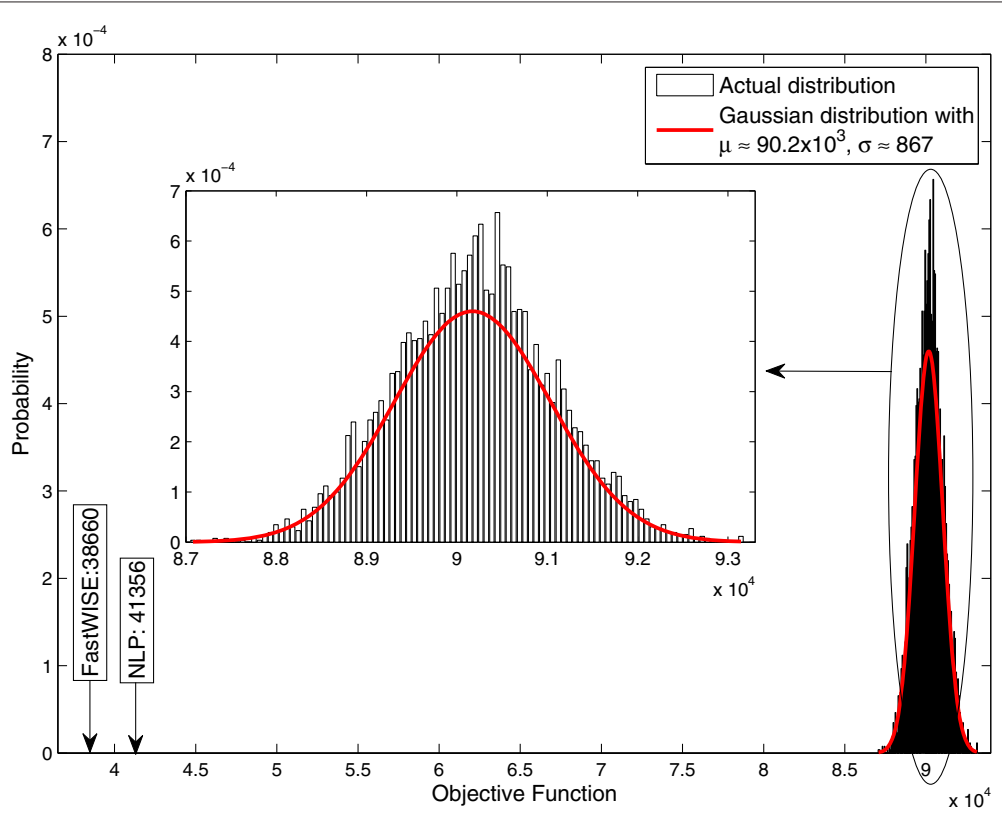

Figure 6 Distribution of feasible MC experiments and its comparison with FastWISE and NLP. 


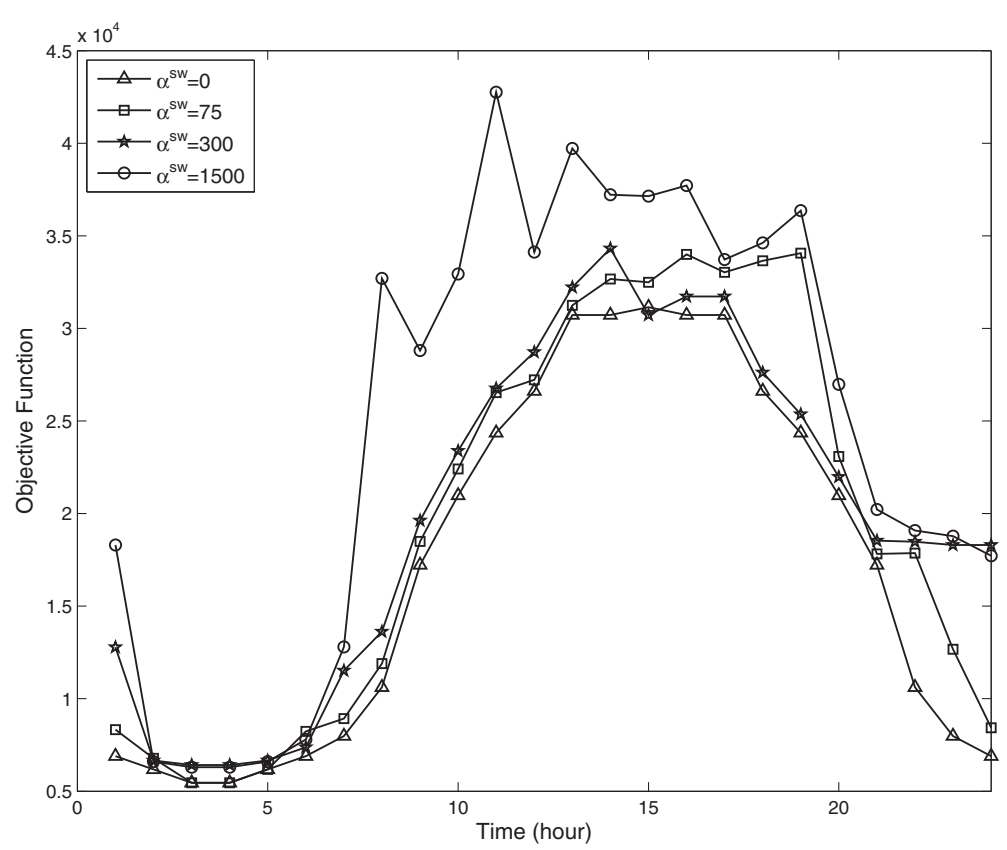

Figure 7 Effect of $\alpha^{\mathrm{sw}}$ on the objective function given in (6).

energy-efficient solutions. Therefore, the network operators should delicately choose this parameter according to their requirements.

Figures 9 and 10 depict the coverage of FastWISE after each phase during light and heavy traffic conditions. In the initialization phase, FastWISE tries to fill the gaps without violating the capacity constraints as seen in Figures 9a and 10a. Then, in the iteration phase, it turns on the BSs with appropriate power levels in order to satisfy the coverage constraints as seen in Figures 9b and 10b. Finally, in the validation phase, FastWISE checks the offered loads for each BS and validates that they are not overloaded. If a BS

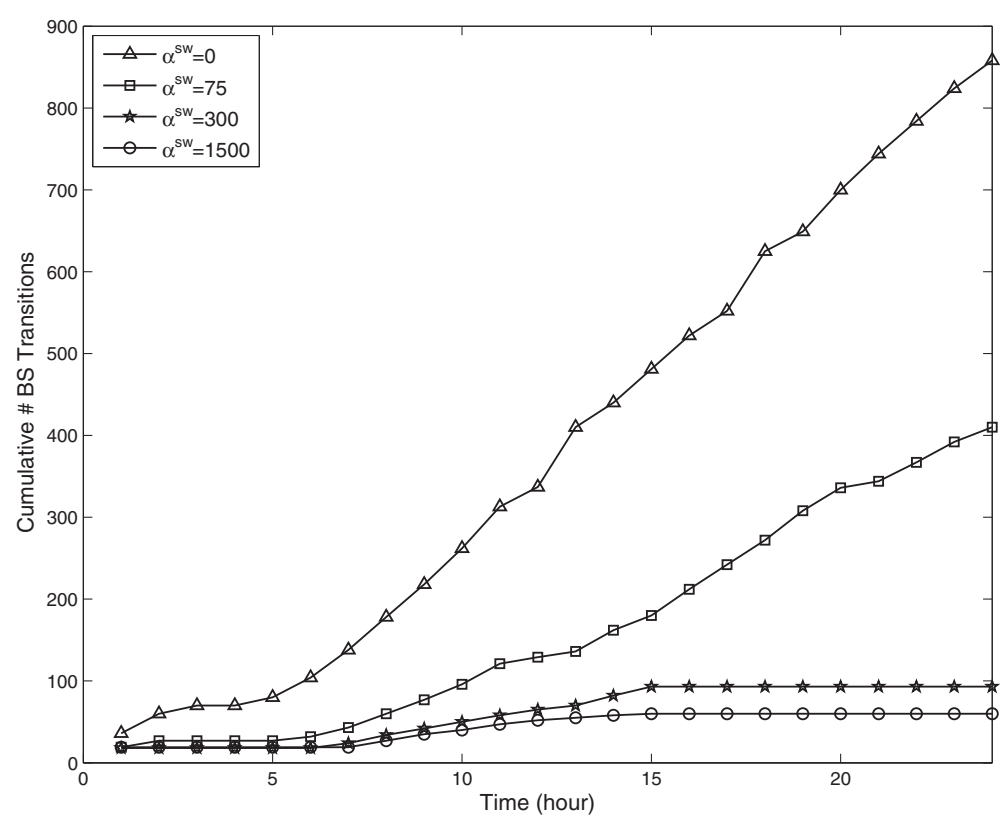

Figure 8 Effect of $\alpha^{\mathrm{sw}}$ on the cumulative number of BS on/off transitions. 

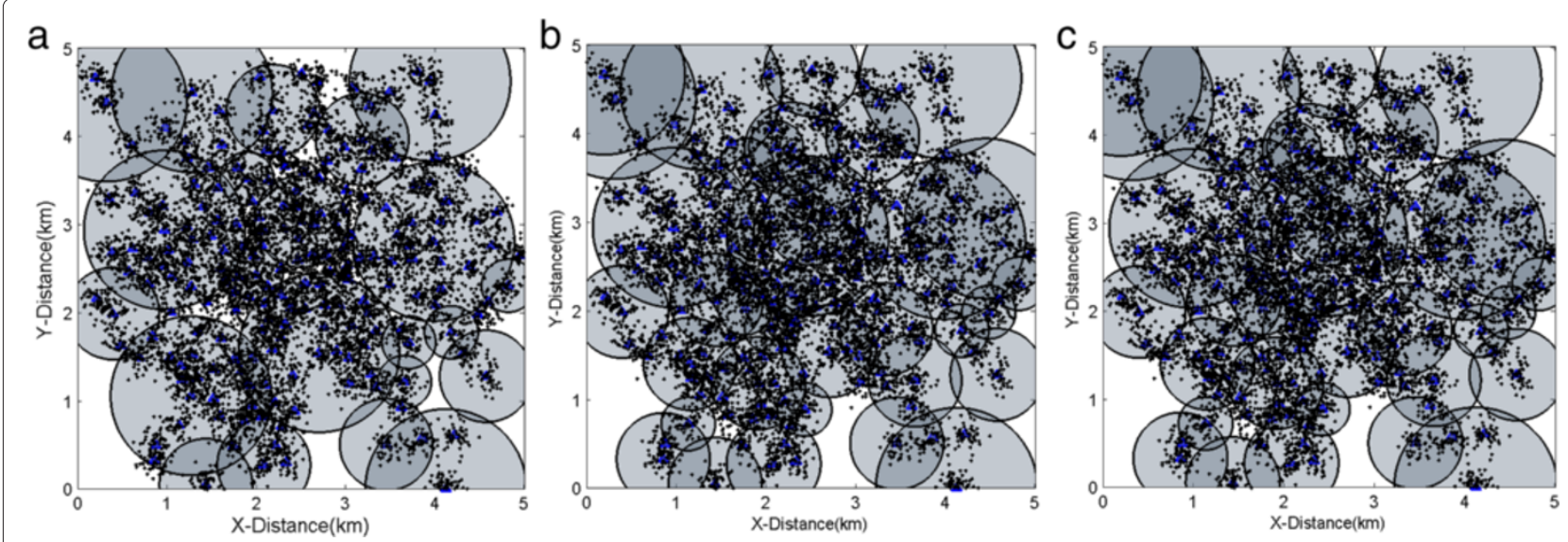

Figure 9 Resulting coverage of FastWISE under light traffic load. (a) After initialization phase (COV cur $\left.=83.06 \%, p^{\text {blck }}=1.7 \times 10^{-3}\right)$. (b) After iteration phase $\left(\right.$ COV $\left.^{\text {cur }}=99.02 \%, p^{\text {blck }} \approx 0\right)$. (c) After validation phase $\left(\right.$ COV cur $\left.=99.02 \%, p^{\text {blck }} \approx 0\right)$.

is overloaded, FastWISE turns the neighboring cell sites to alleviate its load until that particular BS can accommodate the offered traffic without violating the GoS constraints. The resulting coverage after the validation phase is given in Figures 9c and 10c.

\section{Conclusions}

In this work, we focus on saving energy by both turning BSs on/off and adaptively adjusting their transmission power according to the current traffic conditions. To achieve that goal, we formulated a novel nonlinear programming model for the GDBP problem to find the best possible BS topology which minimizes the energy consumption of the network while satisfying a certain level of GoS. Optimization tools can produce optimum results for the small instances of the problem. We derived a greedy heuristic called FastWISE to solve the large realistic size instances of the formulated problem and compared our results with the results of a non-commercial optimization tool and numerous MC experiments. It is shown that our green dynamic BS planning scheme adaptively adjusts to the current traffic load and saves significant amount of energy without violating the GoS constraints such as the probability of blocking and the coverage ratio.

As a future work, we are planning to improve our work to be applied in data-oriented cellular networks. Therefore, the effects of transmission power adjustment on the neighboring cell sites are required to be explored in detail. Another aspect that needs to be taken into consideration is the handoff overhead stemming from frequent topology changes. It would be very useful to implement user association rules integrated with our GDBP solution in order to reduce the handoff rates. Another interesting issue as a future work can be the installation of additional BSs to achieve greener topologies. By this way, a small amount of investment can save significant amount
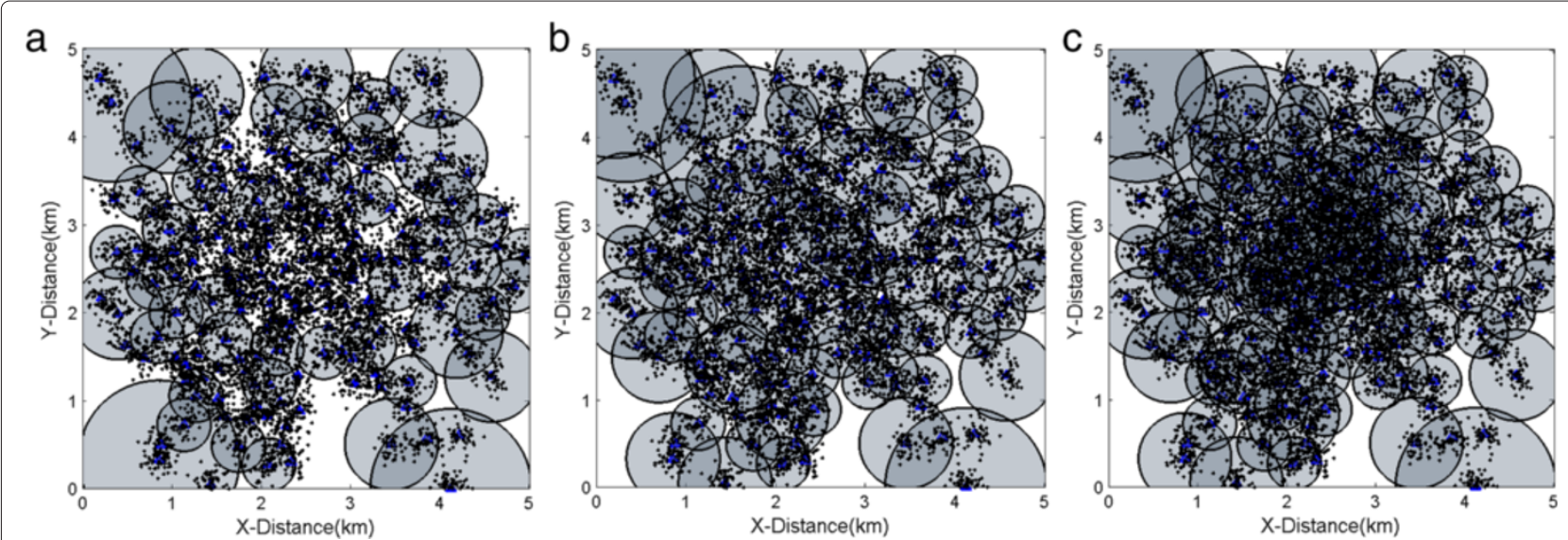

Figure 10 Resulting coverage of FastWISE under heavy traffic load. (a) After initialization phase $\left(\right.$ COV $\left.{ }^{\text {cur }}=49.12 \%, p^{\text {blck }}=16 \times 10^{-3}\right)$. (b) After iteration phase $\left(C^{\text {cur }}=99.1 \%, p^{\text {blck }}=892 \times 10^{-3}\right)$. (c) After validation phase $\left(\right.$ COV $\left.^{\text {cur }}=99.12 \%, p^{\text {blck }}=9 \times 10^{-3}\right)$. 
of energy and become very profitable in the long term. Lastly, we are planning to improve our GDBP scheme to be applied in heterogeneous access networks having different types of BSs in terms of coverage, capacity, and power consumption.

\section{Endnotes}

${ }^{\text {a }}$ Incremental users covered by that particular BS when it is turned on.

${ }^{\mathrm{b}}$ Proper PL is the highest possible PL that a BS can operate without violating the capacity constraint.

${ }^{\mathrm{c}}$ Recall that the BS core power consumption is assumed to be fixed in Section 3.2.

\section{Competing interests}

The authors declare that they have no competing interests.

\section{Acknowledgements}

This work is supported by the Turkish State Planning Organization (DPT) under the TAM Project, number 2007K120610 and by the Turk Telekom Group under the 'Green Dynamic Base Station Planning with Power Adaptation for Wireless Cellular Networks' project.

\section{Author details}

${ }^{1}$ Computer Networks Research Laboratory, Netlab., Department of Computer Engineering, Bogazici University, Bebek, Istanbul 34342, Turkey. ${ }^{2}$ Department of Computer Engineering, Galatasaray University, Ortakoy, Istanbul 34349, Turkey.

Received: 20 August 2013 Accepted: 29 April 2014

Published: 15 May 2014

\section{References}

1. The Climate Group, Global e-Sustainability Initiative (GeSI), Smart 2020: enabling the low carbon economy in the information age. http://www. smart2020.org. Accessed 12 May 2014

2. Ofcom, Understanding the environmental impact of communication systems. http://stakeholders.ofcom.org.uk/market-data-research/other/ technology-research/research/sector-studies/environment. Accessed 6 Nov 2013

3. Cisco, White paper on Cisco Visual Networking Index: forecast and methodology, 2011-2016 (Cisco Systems Inc., San Jose, 2012)

4. Cisco, White paper on Cisco Visual Networking Index: global mobile data traffic forecast update, 2010-2015 (Cisco Systems Inc., San Jose, 2011)

5. S Vadgama, M Hunukumbure, Trends in green wireless access networks, in 2011 IEEE International Conference on Communications Workshops (ICC), Kyoto, 5-9 June 2011, pp. 1-5

6. Australian Geographical Radio Frequency Map. http://maps.spench.net. Accessed 11 Dec 2013

7. Australian Communications and Media Authority. http://www.acma.gov. au. Accessed 5 June 2013

8. E Oh, B Krishnamachari, Energy savings through dynamic base station switching in cellular wireless access networks, in Global Telecommunications Conference (GLOBECOM 2010), IEEE, pp. 1-5, Miami, 6-10 December 2010

9. MA Marsan, L Chiaraviglio, D Ciullo, M Meo, Optimal energy savings in cellular access networks, in IEEE International Conference on Communications Workshops, 2009. ICC Workshops 2009, pp. 1-5, Dresden, 14-18 June 2009

10. K Son, H Kim, Y Yi, B Krishnamachari, Base station operation and user association mechanisms for energy-delay tradeoffs in green cellular networks. Selected Areas Commun. IEEE J. 29(8), 1525-1536 (2011)

11. S Zhou, J Gong, Z Yang, Z Niu, P Yang, Green mobile access network with dynamic base station energy saving, in ACM MobiCom'09, pp. 10-12, Beijing, 20-25 September 2009
12. E Oh, K Son, B Krishnamachari, Dynamic base station switching-on/off strategies for green cellular networks. IEEE Transactions on Wireless Communications. 12(5), 2126-2136 (2013)

13. L Saker, SE Elayoubi, Sleep mode implementation issues in green base stations, in 2010 IEEE 21st International Symposium on Personal Indoor and Mobile Radio Communications (PIMRC), pp. 1683-1688, Istanbul, 26-30 September 2010

14. D Tipper, A Rezgui, P Krishnamurthy, P Pacharintanakul, Dimming cellular networks, in 2010 IEEE Global Telecommunications Conference (GLOBECOM 2010), pp. 1-6, Miami, 6-10 December 2010

15. MA Marsan, M Meo, Energy efficient management of two cellular access networks. SIGMETRICS Perform Eval. Rev. 37, 69-73 (2010)

16. Z Niu, Y Wu, J Gong, Z Yang, Cell zooming for cost-efficient green cellular networks. Commun. Mag. IEEE. 48(11), 74-79 (2010)

17. S Bhaumik, G Narlikar, S Chattopadhyay, S Kanugovi, Breathe to stay cool: adjusting cell sizes to reduce energy consumption, in Proceedings of the First ACM SIGCOMM Workshop on Green Networking. Green Networking '10, pp. 41-46, New Delhi, 30 August - 3 September 2010

18. S Kokkinogenis, G Koutitas, Dynamic and static base station management schemes for cellular networks, in 2012 IEEE Global Communications Conference (GLOBECOM), pp. 3443-3448, Anaheim, 3-7 December 2012

19. LChiaraviglio, D Ciullo, M Meo, MA Marsan, Energy-efficient management of UMTS access networks, in 21st International Teletraffic Congress, 2009. ITC 21 2009, pp. 1-8 (2009)

20. Z Hasan, H Boostanimehr, VK Bhargava, Green cellular networks: A survey, some research issues and challenges. Commun. Surv. Tutor. IEEE. 13(4), 524-540 (2011)

21. F Richter, AJ Fehske, P Marsch, GP Fettweis, Traffic demand and energy efficiency in heterogeneous cellular mobile radio networks, in 2010 IEEE 71st Vehicular Technology Conference (VTC 2010-Spring), pp. 1-6 (2009)

22. MA Marsan, L Chiaraviglio, D Ciullo, M Meo, Switch-off transients in cellular access networks with sleep modes, in 2011 IEEE International Conference on Communications Workshops (ICC), pp.1-6 (2011)

23. L Sun, H Tian, P Zhang, Decision-making models for group vertical handover in vehicular communications. Telecommun. Syst. 50(4), 257-266 (2012)

24. Q Lu, M Ma, Group mobility support in mobile WiMAX networks. J. Netw. Comput. Appl. 34(4), 1272-1282 (2011)

25. D Willkomm, S Machiraju, J Bolot, A Wolisz, Primary users in cellular networks: a large-scale measurement study, in 3rd IEEE Symposium on New Frontiers in Dynamic Spectrum Access Networks, 2008. DySPAN 2008, pp. 1-11 (2008)

26. TS Rappaport, Wireless Communications: Principles and Practice, 2 nd edn, (Prentice Hall, New Jersey, 2002)

27. Basic Open-source Nonlinear Mixed INteger programming(BONMIN). http://www.coin-or.org/Bonmin. Accessed 7 Nov 2013

28. A Modeling Language for Mathematical Programming(AMPL). http:// www.ampl.com. Accessed 7 Nov 2013

29. EnergyAustralia. http://www.energyaustralia.com.au. Accessed 13 Oct 2013

doi:10.1186/1687-1499-2014-77

Cite this article as: Yigitel et al.: Dynamic base station planning with power adaptation for green wireless cellular networks. EURASIP Journal on Wireless Communications and Networking 2014 2014:77. 